\title{
Simulação de um modelo de dispersão de poluente com reação química na camada limite atmosférica
}

\author{
Simulation of a model of dispersion of pollution with chemical reaction in the at- \\ mospheric boundary layer
}

Guilherme Jahnecke Weymar ${ }^{1}$, Daniela Buske ${ }^{2}$, Régis Sperotto de Quadros ${ }^{3}$, Jonas Da Costa Carvalho ${ }^{4}$

${ }^{1}$ Universidade Federal de Pelotas, Pelotas, Brasil

guilhermejahnecke@gmail.com

${ }^{2}$ Universidade Federal de Pelotas, Pelotas, Brasil

danielabuske@gmail.com

${ }^{3}$ Universidade Federal de Pelotas, Pelotas, Brasil

quadros99@gmail.com

${ }^{4}$ Universidade Federal de Pelotas, Pelotas, Brasil

jonascc@yahoo.com.br

\section{Resumo}

Este trabalho apresenta uma representação analítica para um modelo de dispersão de poluentes que considera a reação química, o modelo utiliza a equação de advecção-difusão tridimensional para descrever o campo de concentração na camada limite atmosférica e para representar a reação química que o poluente sofre é incluído um termo fonte na equação. Para resolver o problema utiliza-se o método da Decomposição de Adomian modificado associado com o método 3D-GILTT. O modelo foi aplicado para simular a dispersão e o transporte do $\mathrm{SO}_{2}$ (díóxido de enxofre), poluente produzido da queima do carvão mineral, emitido pela usina termelétrica Presidente Médici, localizada em Candiota/RS. Com a análise dos resultados pode-se observar que as concentrações geradas pelo modelo são satisfatórias e que a abordagem utilizada é uma nova proposta para a descrição do campo de concentração de uma substância.

Palavras-chave: Reação química, equação de advecção-difusão, método da Decomposição de Adomian, método 3D-GILTT.

\section{Abstract}

This work presents an analytical representation for a dispersion model of pollutants that considers the chemical reaction, the model uses the three-dimensional advection-diffusion equation to describe the concentration field in the atmospheric boundary layer and to represent the chemical reaction that the pollutant suffers is included a source term in the equation. To solve the problem we use the modified Adomian Decomposition method associated with the 3D-GILTT method. The model was applied to simulate the dispersion and transportation of the $\mathrm{SO}_{2}$ (sulfur dioxide), a pollutant produced from the burning of mineral coal, emitted by the Presidente Médici thermoelectric plant, located in Candiota / RS. With the analysis of the results it can be observed that the concentrations generated by the model are satisfactory and that the approach used is a new proposal for the description of the concentration field of a substance.

Keywords: Chemical reaction, advection-diffusion equation, Adomian Decomposition method, 3D-GILTT method. 


\section{Introdução}

A poluição atmosférica nas cidades de hoje são em grande parte causadas pela industrialização e o aumento da emissão de poluentes resultantes da queima de combustíveis fósseis por veículos automotores. Nos dias de hoje muitas indústrias possuem em suas instalações sistemas de filtragem e de tratamento dos gases e vapores que são lançados nos processos que a indústria realiza. E para esse tratamento é importante que se tenha um monitoramento da qualidade do ar.

Desta forma, sempre que há interesse em trabalhar e estudar modelos de poluição do ar e dispersão de poluentes, necessitase antes de tudo levar em conta a atmosfera em que o transporte e difusão de poluentes ocorrem, bem como seus procesos de remoção e transformação química.

Os modelos matemáticos são instrumentos particularmente úteis para o entendimento dos fenômenos que controlam o transporte, a dispersão e a transformação físico-química dos poluentes lançados na atmosfera. Os modelos permitem uma avaliação dos níveis de concentração em torno da fonte poluidora, podem ser utilizados para evitar eventos críticos de poluição, além de discriminar os efeitos de várias fontes e de vários poluentes (Moreira e Tirabassi, 2004).

Para descrever o campo de concentração de um poluente na camada limite atmosférica(CLA), utiliza-se a equação de advecção-difusão (Pasquill e Smith, 1983), que é obtida através da parametrização dos fluxos turbulentos na equação da continuidade. Nesta equação é acrescentado um termo de reação fotoquímica (processo de primeira ordem), este termo representará a reação fotoquímica que transforma o $\mathrm{SO}_{2}$.

Neste trabalho, apresenta-se uma representação analítica para a equação de advecção-difusão tridimensional aplicada à dispersão de poluentes que sofrem reações fotoquímicas na camada limite atmosférica, e para resolver este problema utiliza-se o método da Decomposição de Adomian modificado (Adomian, 1994) associado com o método 3D-GILTT (Buske et al., 2012).

\section{Metodologia}

\subsection{Descrição do termo de reação química}

A radiação ultravioleta fornece a energia necessária para as reações químicas que acontecem na atmosfera, de acordo com Shirmer e Lisboa (2008) as principais espécies sulfuradas que sofrem reações químicas são: $\mathrm{COS}_{2} \mathrm{CS}_{2}, \mathrm{SO}_{2} \mathrm{SO}_{4}^{(-2)}\left(\mathrm{CH}_{3}\right)_{2}$ $\mathrm{S}, \mathrm{H}_{2} \mathrm{~S}$. Muitos desses compostos entram na atmosfera como resultado da atividade humana, sendo $\mathrm{SO}_{2}$ o mais abundante, decorrente da queima de combustíveis fósseis (carvão) e atividades industriais. Pretende-se inserir um termo fonte na equação de advecção-difusão, que caracterize a reação fotoquímica que o poluente dióxido de enxofre $\left(\mathrm{SO}_{2}\right)$ sofre na troposfera. Sendo assim, considera-se a seguinte reação fotoquímica:

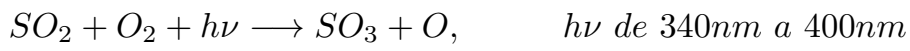

A fotodissociação do $\mathrm{SO}_{2}$ equação (1), pode ser descrita como um processo de primeira ordem (Finlayson-Pitts e Pitts, 2000b), representado por:

$$
\frac{d \bar{c}_{\mathrm{SO}_{2}}}{d t}=-J_{\mathrm{SO}_{2}} \bar{c}_{\mathrm{SO}_{2}}
$$

sendo $\bar{c}_{\mathrm{SO}_{2}}$ a concentração média do dióxido de enxofre, $\mathrm{J}_{\mathrm{SO}_{2}}$ é a frequência de fotólise de $\mathrm{SO}_{2}$, que segundo Jacobson (2005) pode-se calcular essa taxa da seguinte maneira:

$$
J_{S_{2}}=\int_{0}^{\infty} \Phi_{S O_{2}}(\lambda) \sigma_{S O_{2}}(\lambda) F(\lambda) \mathrm{d} \lambda
$$

onde $F$ é o fluxo actínio integrado esfericamente, $\sigma_{\mathrm{SO}_{2}}$ é a seção de choque de absorção e $\Phi_{\mathrm{SO}_{2}}$ é o rendimento quântico do gás dióxido de enxofre.

A radiação que passa através da atmosfera sofre perdas, ou seja, o fluxo actínio espectral é reduzido pela presença dos gases e aerossóis presentes na atmosfera, devido a absorção e espalhamento desses. Esta lei pode ser expressa pela lei de Beer-Lambert (Finlayson-Pitts e Pitts, 2000a):

$$
\mathrm{d} F_{\lambda}=-k_{\lambda} F_{\lambda} d s
$$

no qual $F_{\lambda}$ é a radiância espectral ao longo de um caminho na direção $s$ e $k_{\lambda}$ é chamado de coeficiente de extinção. O coeficiente de extinção pode ser expresso como: $k_{\lambda}=\sigma(\lambda) \rho(s)$, onde $\rho(s)$ é a densidade dos gases radiativamente ativos (que podem variar ao longo do caminho $s$ ) e $\sigma$ é a seção de choque de extinção no comprimento de onda $\lambda$. A extinção é o somatório das seções de choque de absorção e espalhamento $\sigma=\sigma_{a}(\lambda)+\sigma_{e}(\lambda)$. Considera-se apenas a absorção dos gases, $\log \sigma(\lambda)=\sigma_{a}(\lambda)$, e, portanto:

$$
k_{\lambda}=\sum_{i=1}^{N} \sigma_{a, i}(\lambda) \rho_{i}(s),
$$


nos quais $\sigma_{a, i}$ e $\rho_{i}(s)$ são a seção de choque de absorção e a densidade na altura $s$ do gás $i$, respectivamente $(N$ representa o número de gases que compõem a atmosfera limpa).

A atmosfera é composta na sua maior parte pelos gases de nitrogênio (78\%) e oxigênio (21\%), também se encontram em pequenas proporções argônio, dióxido de carbono, água e ozônio, outros gases estão presentes na composição da atmosfera. porém em pequeníssimas proporções.

Na região de espectro de $0,2 \mu \mathrm{m}$ a $0,7 \mu \mathrm{m}$, segundo Mélières e Maréchal (2010) a maior parte da radiação solar nessa região é absorvida pelos gases $\mathrm{O}_{2}$ e $\mathrm{O}_{3}$. Então, para o cálculo de radiação solar que atinge a CLA, considera-se apenas a absorção do oxigênio e ozônio: $k_{\lambda} \approx \sigma_{a, O_{2}}(\lambda) n_{O_{2}}(s)+\sigma_{a, O_{3}}(\lambda) n_{O_{3}}(s)$.

As seções de choque de absorção do oxigênio e ozônio foram parametrizadas utilizando os dados de Rudek et al. (2013) e Finlayson-Pitts e Pitts (2000b) e um ajuste de curvas com o método dos mínimos quadrados.

Para determinar a densidade do oxigênio e ozônio usou-se os dados disponíveis em Schlatter (2009) e construiu-se a função densidade de gases na atmosfera em função da altitude. Assim, a solução para a equação de Beer-Lambert é:

$$
F_{\lambda}=F_{0}(\lambda) e^{-\int_{0}^{z}\left[\sigma_{a, O_{2}}(\lambda) \rho_{O_{2}}(s)+\sigma_{a, O_{3}}(\lambda) \rho_{O_{3}}(s)\right] \mathrm{d} s} .
$$

A Figura 1 representa a radiação solar que atinge a CLA, utilizou-se os dados de Thekaekara (1973) para obter a radiação que atinge o topo da atmosfera $\left(F_{0}(\lambda)\right)$.

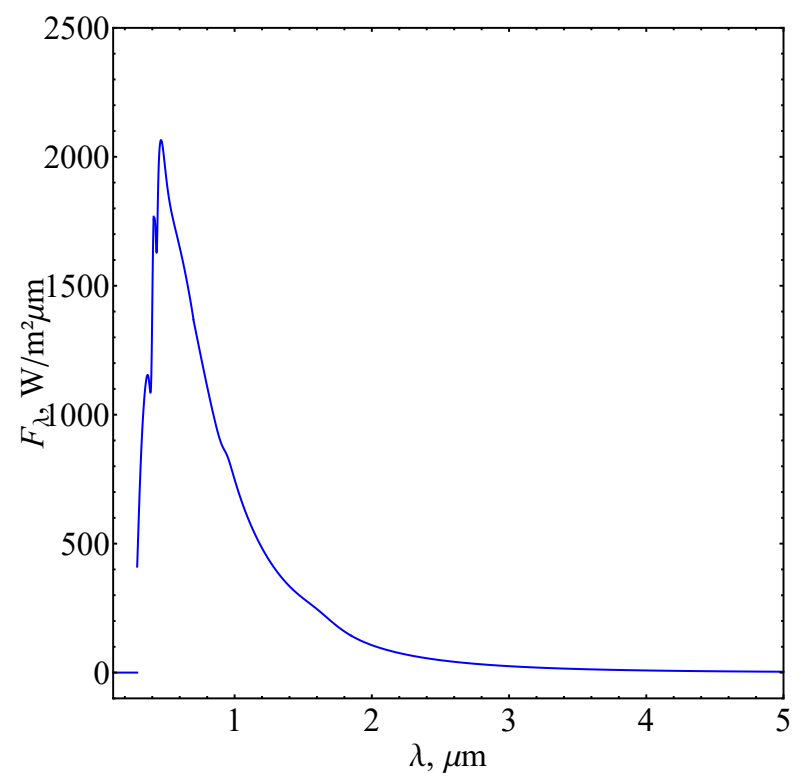

Figura 1: Radiação solar que atinge a CLA.

Uma vez determinado o fluxo actínio que atinge a CLA, deve-se determinar a seção de choque de absorção e o rendimento quântico de $\mathrm{SO}_{2}$ para o cálculo da taxa de fotólise desse gás. De maneira análoga é obtida a seção de choque de absorção de $\mathrm{SO}_{2}$ (Rudek et al., 2013). E para o rendimento quântico desse elemento utilizou-se os valores em Sethi (1971).

\subsection{Solução do problema}

Para modelar o campo de concentração do dióxido de enxofre será utilizada a equação de advecção-difusão tridimensional transiente acrescentada do termo de reação fotoquímica (processo de primeira ordem), este termo representará a fotodissociação do elemento $\mathrm{SO}_{2}$ :

$$
\frac{\partial \bar{c}}{\partial t}+\vec{v} \cdot \nabla \bar{c}=\nabla \cdot(\mathbb{K} \cdot \nabla) \bar{c}-J_{S O_{2}} \bar{c}
$$

sendo $\bar{c}$ a concentração média do poluente dióxido de enxofre, $\vec{v}=(\bar{u}, \bar{v}, \bar{w})$ a velocidade média do vento, $\mathbb{K}$ a matriz dos coeficientes de difusão $\mathbb{K}=\operatorname{diag}\left(K_{x}, K_{y}, K_{z}\right)$. O domínio de interesse é um cubo com dimensões $L_{x}, L_{y}$ e $h$, na qual $h$ é a altura da camada limite planetária. A equação (7) está sujeita às condições de contorno de fluxo nulo nas faces do cubo, concentração inicial nula (em $t=0)$ e a fonte de emissão é aproximada por uma fonte pontual $\bar{u} \bar{c}(0, y, z, t)=Q \delta\left(y-y_{0}\right) \delta\left(z-H_{s}\right)$ sendo $Q$ e $\left(0, y_{0}, H_{s}\right)$ a taxa de emissão e a posição da fonte, nessa ordem. 
Para resolver este problema, aplica-se inicialmente o método da Decomposição de Adomian modificado, ou seja, expande-se a concentração média do poluente em série:

$$
\bar{c}(x, y, z, t)=\sum_{n=0}^{\infty} \overline{c_{n}}(x, y, z, t)
$$

Substituindo a equação (8) na equação (7) e truncando a série (8) de forma que a expansão convirja. Dessa forma constrói-se um sistema recursivo para $\bar{c}$

$$
\begin{gathered}
\frac{\partial \overline{c_{0}}}{\partial t}+\vec{v} \cdot \nabla \overline{c_{0}}=\nabla \cdot(\mathbb{K} \cdot \nabla) \overline{c_{0}} \\
\frac{\partial \overline{c_{n}}}{\partial t}+\vec{v} \cdot \nabla \overline{c_{n}}=\nabla \cdot(\mathbb{K} . \nabla) \overline{c_{n}}-J_{S O_{2}} \overline{c_{n-1}}
\end{gathered}
$$

onde $\overline{c_{0}}$ e $\overline{c_{n}}$ são respectivamente o primeiro e o termo genérico da expansão da solução em série. A equação (9) está sujeita às condições de contorno e inicial do problema, e para a equação (10) as condições de contorno e iniciais são nulas.

Assim, para resolver as equações (9) e (10) utiliza-se o método 3D-GILTT (Buske et al., 2012) que pode ser resumido nos seguintes passos: aplica-se o método espectral na variável y, transformando as equações (9) e (10) em um sistema de equações advectivas-difusivas bidimensionais transientes, que são resolvidas pela técnica GILTT; posteriormente aplica-se a transformada de Laplace na variável temporal resultando num problema estacionário bidimensional e aplicando novamente o método espectral, agora na variável z, obtém-se uma equação diferencial ordinária de primeira ordem, que é facilmente resolvida. Desta forma estão bem determinados e assim a concentração média do poluente é conhecida.

\section{Resultados numéricos}

Segundo Moreira et al. (2007), os modelos de transporte e difusão de poluentes na atmosfera devem prescindir de um estudo sobre suas capacidades de representarem corretamente situações reais, para a utilização correta destes.

Desta forma, aplicou-se o modelo para simular as concentrações superficiais de $\mathrm{SO}_{2}$ devido à emissão da usina termelétrica Presidente Médici. A usina é uma importante fonte de liberação de $\mathrm{SO}_{2}$ na região. Esta é uma fonte pontual e contínua, emitindo poluentes a uma taxa de aproximadamente $0,7 \mathrm{kgs}^{-1}$.

Os dados meteorológicos de entrada do modelo foram coletados durante um experimento de campo realizado no inverno de 1999, em um ponto localizado a $5 \mathrm{~km}$ na direção leste da fonte. Esses dados são médias horárias de temperatura, velocidade e direção do vento, valor líquido do fluxo de radiação e umidade relativa (Arbage et al., 2006).

A campanha experimental foi realizada entre 23 de agosto e 01 de setembro de 1999. Simulou-se apenas os dias, 28,29 e 30 de agosto de 1999. As parametrizações da turbulência e o perfil de vento utilizados neste trabalho, podem ser encontrados em Buske et al. (2012).

Na Tabela 1, apresentam-se os índices estatísticos descrito por Hanna e Paine (1989), em que os melhores resultados são valores próximos de 0 para os índices NMSE (erro quadrático médio normalizado), FB (fração de inclinação) e FS (desvio fracional padrão), e próximo de 1 para o índice COR (coeficiente de correlação). Note que os subíndices o e p referem-se as quantidades observadas e preditas, respectivamente, e indica os valores médios. Pode-se observar que resultados obtidos pelo modelo são razoáveis, pois no modelo proposto foram feitas algumas aproximações como as parametrizações para os coeficientes de difusão turbulenta, o perfil de vento e a reação fotoquímica, também é importante destacar que são poucos dados analisados.

Tabela 1: Avaliação estatística do modelo.

\begin{tabular}{cc}
\hline Índices Estatísticos & Modelo Proposto \\
\hline$N M S E=\frac{\left.\overline{\left(C_{o}-C_{p}\right.}\right)^{2}}{\overline{C_{o} C_{p}}}$ & 0,470 \\
\hline$C O R=\frac{\overline{\left(C_{o}-\overline{C_{o}}\right)\left(C_{p}-\overline{C_{p}}\right)}}{\frac{\sigma_{o} \sigma_{p}}{C_{o}}}$ & 0,572 \\
\hline$F B=\frac{\overline{C_{p}}}{0,5\left(\overline{C_{o}}+\overline{C_{p}}\right)}$ & 0,421 \\
\hline$F S=2 \frac{\sigma_{0}-\sigma_{p}}{\sigma \sigma_{0}+\sigma_{p}}$ & $-0,254$ \\
\hline
\end{tabular}

Na Tabela 2 apresentam-se os resultados numéricos que mostram o número de termos adequados da solução em série (8), para alcançar os resultados com uma precisão de $10^{-4}$. Para isso, é apresentado a Tabela 2 considerando-se, sucessivamente, um, dois, três, quatro e cinco termos na solução em série. Observa-se que a precisão desejada, para o problema resolvido, é atingida com 3 termos da solução em série, para as concentrações geradas com o experimento de Candiota. 
Tabela 2: Concentrações geradas pelo modelo, variando o número de termos da expansão em série (8).

\begin{tabular}{|c|c|c|}
\hline Observação & Número de termos do Método da Decomposição (série (8)) & $\bar{c}(x, y, z, t)\left[\mu g / m^{3}\right]$ \\
\hline \multirow{5}{*}{1} & $\overline{c_{0}}$ & 56,56927 \\
\hline & $\overline{c_{0}}+\overline{c_{1}}$ & 56,48536 \\
\hline & $\overline{c_{0}}+\overline{c_{1}}+\overline{c_{2}}$ & 56,48529 \\
\hline & $\overline{c_{0}}+\overline{c_{1}}+\overline{c_{2}}+\overline{c_{3}}$ & 56,48529 \\
\hline & $\overline{c_{0}}+\overline{c_{1}}+\overline{c_{2}}+\overline{c_{3}}+\overline{c_{4}}$ & 56,48529 \\
\hline \multirow{5}{*}{2} & $\overline{c_{0}}$ & 7,12038 \\
\hline & $\overline{c_{0}}+\overline{c_{1}}$ & 7,12890 \\
\hline & $\overline{c_{0}}+\overline{c_{1}}+\overline{c_{2}}$ & 7,12890 \\
\hline & $\overline{c_{0}}+\overline{c_{1}}+\overline{c_{2}}+\overline{c_{3}}$ & 7,12890 \\
\hline & $\overline{c_{0}}+\overline{c_{1}}+\overline{c_{2}}+\overline{c_{3}}+\overline{c_{4}}$ & 7,12890 \\
\hline \multirow{5}{*}{3} & $\overline{c_{0}}$ & 107,56257 \\
\hline & $\overline{c_{0}}+\overline{c_{1}}$ & 107,43300 \\
\hline & $\overline{c_{0}}+\overline{c_{1}}+\overline{c_{2}}$ & 107,43290 \\
\hline & $\overline{c_{0}}+\overline{c_{1}}+\overline{c_{2}}+\overline{c_{3}}$ & 107,43290 \\
\hline & $\overline{c_{0}}+\overline{c_{1}}+\overline{c_{2}}+\overline{c_{3}}+\overline{c_{4}}$ & 107,43290 \\
\hline \multirow{5}{*}{4} & $\overline{c_{0}}$ & 7,85755 \\
\hline & $\overline{c_{0}}+\overline{c_{1}}$ & 7,85756 \\
\hline & $\overline{c_{0}}+\overline{c_{1}}+\overline{c_{2}}$ & 7,85756 \\
\hline & $\overline{c_{0}}+\overline{c_{1}}+\overline{c_{2}}+\overline{c_{3}}$ & 7,85756 \\
\hline & $\overline{c_{0}}+\overline{c_{1}}+\overline{c_{2}}+\overline{c_{3}}+\overline{c_{4}}$ & 7,85756 \\
\hline \multirow{5}{*}{5} & $\overline{c_{0}}$ & 112,09137 \\
\hline & $\overline{c_{0}}+\overline{c_{1}}$ & 111,93925 \\
\hline & $\overline{c_{0}}+\overline{c_{1}}+\overline{c_{2}}$ & 111,93911 \\
\hline & $\overline{c_{0}}+\overline{c_{1}}+\overline{c_{2}}+\overline{c_{3}}$ & 111,93911 \\
\hline & $\overline{c_{0}}+\overline{c_{1}}+\overline{c_{2}}+\overline{c_{3}}+\overline{c_{4}}$ & 111,93911 \\
\hline
\end{tabular}

\section{Conclusão}

Neste trabalho apresentamos uma nova representação analítica para a solução da equação de advecção-difusão para o problema de dispersão de um poluente e os resultados apresentados pela metodologia proposta, mostraram-se satisfatórios e com poucos termos na expansão do método de Adomian, a concentração não varia mais. Importante destacar que o método da decomposição de Adomian pode ser aplicado em equações diferenciais parciais não-lineares, desta forma o termo de reação química poderia ser representado por uma reação de ordem superior.

Pode-se dizer que a solução apresentada é analítica exceto pelo truncamento dos somatórios, a inversa de Laplace no tempo e a discretização dos coeficientes turbulentos.

Para trabalhos futuros pretende-se aplicar esta metodologia a outros experimentos em que o poluente analisado sofra reações químicas.

\section{Agradecimentos}

Os autores agradecem ao CNPq e FAPERGS pelo apoio financeiro dado a este trabalho.

\section{Referências}

Adomian, G. (1994). Solving frontier problem of physics: the decomposition method. Springer.

Arbage, M. C. A., Degrazia, G. A., Moraes, O. L. (2006). Simulação euleriana da dispersão local da pluma de poluente atmosférico de candiota-rs. Brasileira de Meteorologia, 21, 153-160.

Buske, D., Vilhena, M. T., Tirabassi, T., Bodmann, B. (2012). Air pollution steady-state advection-diffusion equation: the general three-dimensional solution. Journal of Environmental Protection, 4, 1-10. 
Finlayson-Pitts, B., Pitts, J. (2000a). Spectroscopy and photochemistry fundamentals. Em: Chemistry of the Upper and Lower Atmosphere, pp. 43-85.

Finlayson-Pitts, B. J., Pitts, J. N. (2000b). Photochemistry of important atmospheric species. Em: Chemistry of the Upper and Lower Atmosphere, pp. 86-129.

Hanna, S., Paine, R. (1989). Hibrid plume dispersion model (hpdm) development and evaluation. Journal of Applied Meteorology, $28,206-224$

Jacobson, M. (2005). Fundamentals of Atmospheric Modeling, $2^{\circ}$ edn. Cambridge University Press.

Mélières, M., Maréchal, C. (2010). Climate Change: Past, Present and Future. CRDP de l'Académic de Grenoble.

Moreira, D., Tirabassi, T. (2004). Modelo matemático de dispersão de poluentes na atmosfera: um instrumento técnico para gestão ambiental. Ambiente \& Sociedade, 7, 169-171.

Moreira, D. M., Vilhena, M. T., Carvalho, J. d. C. (2007). Tritium dispersion simulation in the atmosphere from angra i nuclear power plant. International Journal of Nuclear Energy Science and Technology, 3, 118-130.

Pasquill, F., Smith, F. (1983). Atmospheric Diffusion. Halsted Press.

Rudek, H. K., Moortgat, G., Sander, R., Sörensen, R. (2013). The mpi-mainz uv/vis spectral atlas of gaseous molecules of atmospheric interest. Earth System Science Data, pp. 365-373.

Schlatter, T. (2009). Atmospheric composition and vertical structure. Environmental Impact and Manufacturing, pp. 1-53.

Sethi, D. (1971). Photo-oxidation of sulfur dioxide. Air Pollution Control Association, pp. 418-420.

Shirmer, W. N., Lisboa, H. d. M. (2008). Química da atmosfera: Constituintes naturais, poluentes e suas reações. Tecno-Lógica, $46,12-37$.

Thekaekara, M. (1973). Solar energy motion in space (semis). Symposium Solar Radiation Measurements and Instrumentation, pp. 414-442.

Guilherme Jahnecke Weymar

Universidade Federal de Pelotas UFPEL E-mail: guilhermejahnecke@gmail.com

Daniela Buske

Universidade Federal de Pelotas UFPEL

E-mail: danielabuske@gmail.com

Régis Sperotto de Quadros

Universidade Federal de Pelotas UFPEL E-mail: quadros99@gmail.com

Jonas da Costa Carvalho

Universidade Federal de Pelotas UFPEL E-mail: jonascc@yahoo.com.br 